\title{
How Important Is On-Site Adequacy Assessment for Thyroid FNA? An Evaluation of 883 Cases
}

\author{
Weijian Zhu, M.D., Ph.D. and Claire W. Michael, M.D.*
}

\begin{abstract}
Editor's Note: This article is in response to a very important practical problem surrounding FNA of the thyroid. This procedure can be performed in a doctor's office, an outpatient FNA clinic, a radiological suite, intraoperatively, and in various other settings. The person doing the procedure also can be a number of specialists: cytopathologists, endocrinologists, general surgeons, head and neck physicians, and severa1 other practitioners. Almost everyone agrees that immediate assessment has a role in this procedure. Exactly what role that is and how important its impact constitutes the purpose of this article.
\end{abstract}

Immediate adequacy assessment (IADA) during fine-needle aspiration (FNA) is not universal and the optimal number of passes has not been well determined. The aim of this study was to evaluate the nondiagnostic rates (NDR) with and without the IADA for thyroid aspirates. Subsequent cytological and surgical follow-up were reviewed for nondiagnostic cases. In addition, we evaluated the number of passes performed in each FNA to determine the optimal number.

Retrospective analysis of NDR was performed on 883 thyroid FNA specimens retrieved through a Computer SNOMED Search from our files between January 2001 to December 2003. For FNAs with IADA, one Diff-Quick and one fixed smear for each pass were prepared, and the needle was rinsed in CytoLyt solution for a ThinPrep andlor a cell-block. FNAs without IADA were received in CytoLyt solution, from which a ThinPrep and a cell-block were prepared for each case.

Department of Pathology, University of Michigan Hospitals, Ann Arbor, Michigan

*Correspondence to: Claire W. Michael, M.D., Department of Pathology, University of Michigan Hospitals, 1500 E Medical Center Drive, Room 2G332 University Hospital, Ann Arbor, MI 48109-0054.

E-mail: clairemi@med.umich.edu

Received 2 June 2006; Accepted 7 July 2006

DOI 10.1002/dc.20552

Published online in Wiley InterScience (www.interscience.wiley.com).
Of the total 883 cases, 443 were performed with IADA, of which 417 cases were diagnostic. The remaining 440 cases were performed without IADA, of which 300 cases were diagnostic. NDR for IADA was $5.9 \%$ (26 cases-group-I) compared to $31.8 \%$ (140 cases-group-II) without IADA. In group-I, 5 cases were followed-up by repeat FNA, 10 cases by surgical resection, and 11 cases received no tissue follow-up. In group-II, 23 cases were followed-up by repeat FNA, 36 by surgical resection, and 82 cases received no tissue follow-up. Interestingly, follow-up in group-I did not reveal any missed malignancy, while that in group-II resulted in a malignant diagnosis in $13.8 \%$ (8 cases). We also found that the optimal number of passes with least NDR was 4-6 passes. NDR was 25\% for $<3$ passes, $11 \%$ for 4 passes, $5.2 \%$ for 5 passes, $1.4 \%$ for 6 passes, and $2.5 \%$ for 7 passes or more. IADA significantly reduces the NDR and increases the sample adequacy for diagnosis. Optimal number of passes is 4-6 passes, and additional passes did not improve the diagnostic rate. Our study also emphasizes the significance of repeat FNA or histological follow-up for nondiagnostic cases, especially for those without IADA. Diagn. Cytopathol. 2007; 35:183-186. ○ 2007 Wiley-Liss, Inc.

Key Words: thyroid; fine-needle aspirate; immediate adequacy assessment; nondiagnostic rate

Thyroid lesions (TLs) are a common clinical problem. Fine-needle aspiration (FNA) of the thyroid is now a widely accepted, simple diagnostic method, that proved to be a safe, efficient, reliable, and cost-effective diagnostic procedure to triage TLs for the surgical excision or conservative management. ${ }^{1,2}$ While immediate adequacy assessment (IADA) during fine-needle aspiration (FNA) could be the key for its diagnostic success, it is not universally applied. $^{3-7}$ Our study was designed to determine whether onsite adequacy assessment significantly decreases the nondiagnostic rate (NDR) in the thyroid FNA. Since the management of initial nondiagnostic cases has not been well established and nondiagnostic FNAs of thyroid nod- 
ules remain a significant clinical dilemma, ${ }^{8-11}$ we also investigated the significance of performing a repeat biopsy for those initial nondiagnostic cases by subsequently reviewing cytological and surgical following up. In addition, knowledge of the optimal number of fine needle passes could impact diagnostic accuracy, which has not been well determined. ${ }^{2,7}$ As the second purpose of this study, we evaluated the number of passes per aspirate to determine the optimal number of FNA passes required to obtain an accurate cytologic diagnosis.

\section{Methods}

Retrospective analysis of NDR was performed on 883 thyroid FNA specimens retrieved through a Computer SNOMED Search from our files from January 2001 to December 2003 at University of Michigan Hospitals. For FNAs with IADA of cytopathology, one Diff-Quick and one fixed smear for each pass were prepared and the needle was rinsed in CytoLyt ${ }^{\circledR}$ solution for a ThinPrep and/ or a cell-block. Immediate cytologic examination was performed by a cytopathology fellow and/or cytotechnologists and a faculty. When immediate cytologic assessment was not done, the FNA material was received in CytoLyt solution, from which a ThinPrep and a cell-block were prepared for each case.

The nondiagnostic rate was defined as the number of inadequate cases expressed as a percentage of the total number of cases. Inadequate cases were those that were unsatisfactory for reporting/or unable to offer any diagnosis for reasons including either paucity of cells or any other technical reason such as air-drying, crush artifact, obscuring blood, cells being trapped in fibrin clots and so on. Some initially nondiagnostic cases were followed up with repeat FNAs or biopsies. The statistical significance of the findings was analyzed using Student's $t$ or $\chi^{2}$ tests; $P$ values of 0.05 or less were considered significant.

\section{Results}

The total numbers of FNA cases and percentages [nondiagnostic rate (NDR)] of nondiagnostic cases are given in Table I. The total number of inadequate samples was $166 / 883$ cases, giving an overall NDR of $18.8 \%$. A total of 883 cases were divided into the two main categories, with and without immediate adequacy assessment (IADA) by cytopathologists. Of the 443 with IADA by cytopathologists (group I), 26 cases were nondiagnostic, giving a nondiagnostic rate of $5.9 \%$. In contrast, of the 440 cases without immediate adequacy assessment (Non-IADAgroup II), there were 140 nondiagnostic cases resulting in a nondiagnostic rate of $31.8 \%$. On site evaluation by cytopathologists significantly reduced the NDR of thyroid FNAs $(P<0.001)$. Table II summarizes the distribution of FNA diagnoses for the diagnostic aspirates in the two
Table I. Nondiagnostic Rate in Thyroid Aspirates With and Without Immediate Cytological Assessment

\begin{tabular}{lccc}
\hline & FNAs & $\begin{array}{c}\text { Nondiagnostic } \\
\text { cases }\end{array}$ & $\begin{array}{c}\text { Nondiagnostic } \\
\text { rate (\%) }\end{array}$ \\
\hline IADA & 443 & 26 & $5.9^{*}$ \\
Non-IADA & 440 & 140 & $31.8^{*}$ \\
Total & 883 & 166 & 18.8 \\
\hline$*<0.001$. & & &
\end{tabular}

Table II. Cytological Diagnosis in Thyroid Aspirates

\begin{tabular}{lrc}
\hline & \multicolumn{2}{c}{ Total cases (\%) } \\
\cline { 2 - 3 } & \multicolumn{1}{c}{ IADA } & Non-IADA \\
\hline ATC & $2(0.5)$ & $16(0.3)$ \\
FN & $39(9.4)$ & $3(1.0)$ \\
HT & $9(2.2)$ & $5(1.7)$ \\
HCN & $12(2.9)$ & $0(0)$ \\
ID & $2(0.5)$ & $19(6.4)$ \\
LT & $15(3.6)$ & $3(1.0)$ \\
LYM & $2(0.5)$ & $3(1.0)$ \\
MTC & $1(0.2)$ & $232(77.3)$ \\
NH & $285(68.3)$ & $18(6.0)$ \\
PTC & $50(12.0)$ & $300(100)$ \\
Total & $417(100)$ &
\end{tabular}

ATC, anaplastic thyroid carcinoma; FN, follicular neoplasm; FA, follicular adenoma; HCA, Hurthle cell adenoma; HCCa, Hurthle cell carcinoma; HCN, Hurthle cell neoplasm; HT, Hashimoto's thyroiditis; IADA, immediate adequacy assessment; ID, inflammatory disease; LT, lymphocytic thyroditis; LYM, lymphoma; MTC, medullary thyroid carcinoma; NDR, Nondiagnostic rate; NH, nodular hyperplasia; Non-IADA, nonimmediate adequacy assessment; PTC, papillary thyroid carcinoma.

groups. As in previous reports, the most common diagnosis is nodular hyperplasia, comprising $70 \%$ of cases. There is no significant difference in the distribution of FNA diagnoses between the two groups to account for the difference in the diagnostic rate.

In group I with IADA, 20 of the 443 cases had 3 or less passes per case. Of those 20 cases, 5 cases were nondiagnostic with a nondiagnostic rate of $25 \%$. The NDR was reduced to $11.0 \%(P<0.001)$ by performing 4 FNA passes. Our data also demonstrated a continuous reduction of NDR with the increase in number of FNA passes up to 6 passes. However, additional passes beyond that did not show a significant improvement of NDR (Table III).

Further follow-up by either repeat FNA or surgical resection was available in 14 of the 26 nondiagnostic cases in the FNA group with IADA/group I (Table IV). There was no missed malignant neoplasm identified in the follow-up. In the contrast, of the 140 nondiagnostic cases without IADA/group II, 58 cases had additional followup. Seven initially nondiagnostic cases were diagnosed as papillary thyroid carcinoma (12.1\%), one case as Hurthle cell carcinoma (1.7\%), and two cases as follicular neoplasm $(3.4 \%)$. The overall incidence of malignant neoplasm in the initial nondiagnostic cases is $11 \%(8 / 73)$. About $10-15 \%$ of cases that received a repeat FNA for follow-up remained nondiagnostic. 
Table III. Correlation Between Number of FNA Passes and Nondiagnostic Rates

\begin{tabular}{lccc}
\hline Pass number & $\begin{array}{c}\text { Case } \\
\text { number }\end{array}$ & $\begin{array}{c}\text { Nondiagnostic } \\
\text { cases }\end{array}$ & $\begin{array}{c}\text { Nondiagnostic } \\
\text { rate (\%) }\end{array}$ \\
\hline$\leq 3$ & 20 & 5 & 25.0 \\
4 & 100 & 11 & 11.0 \\
5 & 115 & 6 & 5.2 \\
6 & 67 & 1 & 1.4 \\
7 & 39 & 1 & 2.5 \\
$\geq 8$ & 102 & 2 & 2.0 \\
\hline
\end{tabular}

Table IV. Cytological and Histological Follow-Up of Nondiagnostic Cases

\begin{tabular}{llc}
\hline & IADA & Non-IADA \\
\hline Repeat FNA & & \\
NH & $4(15.5)$ & $14(10.0)$ \\
FN & $0(0)$ & $2(1.4)$ \\
PTC & $0(0)$ & $3(2.1)$ \\
ND & $1(3.8)$ & $3(2.1)$ \\
Histological follow-up & & \\
NH & $7(26.9)$ & $24(17.8)$ \\
HT \& LT & $1(3.8)$ & $3(2.1)$ \\
FA & $2(7.7)$ & $3(2.1)$ \\
HCA & $0(0)$ & $1(0.7)$ \\
HCCa & $0(0)$ & $1(0.7)$ \\
PTC & $0(0)$ & $4(2.9)$ \\
Non-follow-up & $11(42.3)$ & $82(58.6)$ \\
Total & $26(100)$ & $140(100)$ \\
\hline
\end{tabular}

\section{Discussion}

It has been reported that the immediate adequacy assessment of thyroid FNAs has resulted in measurable cost savings by reducing the rate of nondiagnostic aspirates and therefore avoiding the need for repeat FNA. ${ }^{4-7}$ However, other authors claimed that there was no significant difference in cytologic adequacy whether immediate cytologic assessment of aspirated material was performed or not if the FNA is performed under US guidance, and that on-site adequacy evaluation will prolong procedure time, increases patient discomfort, and waste valuable cytological and radiological resources. ${ }^{12}$ Our study demonstrated that the immediate cytologic assessment of thyroid FNAs significantly reduces the number of nondiagnostic cases. The contribution of the cytology assessment significantly reduced the problem of inadequate samples, as also reported by others. ${ }^{3}$ The certainty of having obtained an adequate sample limits the number of passes per FNA and reduces the need for repeat aspiration on the same patient to successfully obtain an adequate sample. This also provides great cost savings as it reduces the cost associated with second hospital visits and additional procedures. ${ }^{6}$ On-site immediate evaluation of FNA specimens also provides the appropriate sample triage for ancillary studies and the ability to issue a preliminary diagnosis, which often facilitates rapid clinical decisions. ${ }^{4-6}$

The immediate assistance by cytology during FNA is not universal, and the optimal number of fine needle passes during the FNA has not been well documented. ${ }^{2,7}$ Although FNA of the thyroid is considered to have a high diagnostic efficiency, it largely depends on the aspirator's and the cytologist's expertise. ${ }^{9,13,14}$ One of the goals in this study was to determine the optimal number of fine needle passes required to reach a diagnosis with certainty. An optimal number would be particularly useful in institutions in which the assistance by cytology is not available. Our study revealed that when the number of needle passes is 3 or less, the NDR could be $25 \%$ or more. NDR was significantly reduced to $11 \%$ with 4 passes performed per thyroid lesions. This suggests that 4 passes/lesion should be the minimum number to reach a reasonable diagnostic rate. Our study also revealed that 6 passes/ nodule demonstrated a very low NDR of $1.5 \%$, which was not improved upon additional passes. Our findings are in agreement with those suggesting 4-6 passes per thyroid nodule as the optimal number of passes needed to maximize diagnostic certainty, in the presence of immediate assessment of specimens by a cytopathologist, ${ }^{9,13,14}$ and confirms that it would provide an optimal yield within the limits of practicality. Multiple passes are particularly important when immediate adequacy assessment is not available.

Although the nondiagnostic rate of FNA is reported to be lower with ultrasound-guided FNA, there are 10-20\% cases continuing to be nondiagnostic. ${ }^{15,16}$ In our study, the incidence of malignancy is $11 \%$ among initial nondiagnostic FNA cases, which is in agreement with the previous reports. ${ }^{10,11}$ There are about $15 \%$ cases remaining to be nondiagnostic despite repeat FNA with IADA. Our data supports the opinion that initial nondiagnostic cases particularly those in the group with no IADA should be repeated due to the high potential of malignancy. $8,10,11$

\section{References}

1. AACE/AME Task Force on Thyroid Nodules. American Association of Clinical Endocrinologists and Associazione Medici Endocrinologi medical guidelines for clinical practice for the diagnosis and management of thyroid nodules. Endocr Pract 2006;12:63-102.

2. Nguyen G-K, Lee MW, Ginsberg J, Wragg T, Bilodeau D. Fineneedle aspiration of the thyroid: An overview. Cytojournal 2005; $2: 12$.

3. Austin JHM, Cohen MB. Value of having cytopathologist present during percutaneous fine-needle aspiration biopsy of lung: Report of 55 cancer patients and meta-analysis of the literature. AJR Am J Roentgenol 1993;160:175-177.

4. Civardi G, Fornari F, Cavanna L, Di Stasi M, Sbolli G, Buscarini L. Value of rapid staining and assessment of ultrasound-guided fine needle aspiration biopsies. Acta Cytol 1988;32:552-554.

5. Ghofrani M, Beckman D, Rimm DL. The value of onsite adequacy assessment of thyroid fine-needle aspirations is a function of operator experience. Cancer 2006;108:110-113.

6. Nasuti JF, Gupta PK, Baloch ZW. Diagnostic value and cost-effectiveness of on-site evaluation of fine-needle aspiration specimens: Review of 5, 688 cases. Diagn Cytopathol 2002;27:1-4. 


\section{ZHU AND MICHAEL}

7. Eedes CR, Wang HH. Cost-effectiveness of immediate specimen adequacy assessment of thyroid fine-needle aspirations. Am J Clin Pathol 2004;121:64-69.

8. Alexander EK, Heering JP, Benson CB, et al. Assessment of nondiagnostic ultrasound-guided fine needle aspirations of thyroid nodules. J Clin Endocrinol Metab 2002;87:4924-4927.

9. Hall TL, Layfield LJ, Phillip A, Rosenthal D. Sources of diagnostic error in fine needle aspiration of the thyroid. Cancer 1989;63:718725 .

10. McHenry CR, Walfish PG, Rosen IB. Non-diagnostic fine needle aspiration biopsy: A dilemma in management of nodular thyroid disease. Am Surg 1993;59:415-419.

11. Chow LS, Gharib H, Goellner JR, van Heerden JA. Nondiagnostic thyroid fine-needle aspiration cytology: Management dilemmas. Thyroid 2001;11:1147-1151.
12. O’Malley ME, Weir MM, Hahn PF, Misdraji J, Wood BJ, Mueller PR. US-guided fine-needle aspiration biopsy of thyroid nodules: Adequacy of cytologic material and procedure time with and without immediate cytologic analysis. Radiology 2002;222:383-387.

13. Gharib H, Goellner J. Fine needle aspiration biopsy of the thyroid: An appraisal. Ann Intern Med 1993;118:282-289.

14. Hamburger B, Gharib H, Melton LJ, Goellner JR, Zinsmeister AR. Fine needle aspiration biopsy of the thyroid nodules. Impact on thyroid practice and cost care. Am J Med 1982;73:381-384.

15. Danese D, Sciacchitano S, Farsetti A, Andreoli M, Pontecorvi A. Diagnostic accuracy of conventional versus sonography-guided fineneedle aspiration biopsy of thyroid nodules. Thyroid 1998;8:15-21.

16. Marqusee E, Benson CB, Frates MC, et al. Usefulness of ultrasonography in the management of nodular thyroid disease. Ann Intern Med 2000; 133:696-700. 